ISSN 1981-416X

Licenciado sob uma Licença Creative Commons

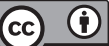

\title{
Jogos digitais na escola e inclusão digital: intervenções para o aprimoramento da atenção e das condições de aprendizagem
}

\section{Digital games at school and digital inclusion: interventions to improve attention and learning conditions}

\author{
Juegos digitales en la escuela e inclusión digital: \\ intervenciones para mejorar la atención \\ y las condiciones de aprendizaje
}

\section{Daniela Karine Ramos ${ }^{[a]}$, Hiago Murilo de Melo ${ }^{[a]}$, João Mattar ${ }^{[b][c] *}$}

\author{
[a] Universidade Federal de Santa Catarina, Florianópolis, SC, Brasil \\ [b] Centro Universitário Internacional Uninter, Curitiba, PR, Brasil \\ [c] Pontifícia Universidade Católica de São Paulo (PUC-SP), São Paulo, SP, Brasil
}

\section{Resumo}

A inclusão digital remete ao acesso e ao uso crítico e criativo das tecnologias digitais em favor da emancipação. Dentre as tecnologias, destacam-se os jogos digitais como alternativas para melhorar as condições de aprendizagem e de letramento digital. Diante disso, este estudo tem como objetivo avaliar se uma intervenção pautada no uso de jogos digitais

*DKR: Doutora em Educação, e-mail: dadaniela@gmail.com HMM: Graduado em Psicologia, e-mail: hmurilodemelo@gmail.com JM: Doutor em Letras, e-mail: joaomattar@gmail.com 
em pequenos grupos em contexto extraclasse pode contribuir com o aprimoramento do desempenho da atenção, visando garantir melhores condições de aprendizagem e inclusão digital. Para tanto, realizou-se uma pesquisa quase experimental de abordagem mista com uma amostra por conveniência de 40 crianças com idades entre 7 e 9 anos, divididas em grupo experimental e controle. O primeiro grupo participou de intervenções semanais em pequenos grupos, com base no uso de jogos digitais. Ambos os grupos foram avaliados antes e depois do período das intervenções, por meio da aplicação de um teste de atenção concentrada. As professoras também foram entrevistadas com relação às mudanças percebidas em sala. Os resultados revelaram que o grupo experimental teve um desempenho da atenção no teste significativamente superior ao grupo controle, especialmente na quantidade de erros cometidos, remetendo ao aprimoramento da qualidade da atenção. Além disso, as professoras destacaram a melhora no foco e na finalização das atividades. Desse modo, reforça-se que o uso dos jogos digitais pode contribuir com a inclusão digital e com a melhora das condições de aprendizagem das crianças.

Palavras-chave: Jogos digitais. Aprendizagem. Educação infantil.

\begin{abstract}
Digital inclusion refers to the access and critical and creative use of digital technologies in favor of emancipation. Among the technologies, digital games stand out as alternatives to improve learning conditions and digital literacy. Therefore, this study aims to evaluate if an intervention based on the use of digital games in small groups in school context can contribute to the improvement of the performance of the attention, aiming to guarantee better conditions of learning and digital inclusion. For that, a quasi-experimental, mixed-sample study was conducted with a convenience sample of 40 children aged 7 to 9 years, divided into experimental and control groups. The first group participated in weekly small group interventions based on the use of digital games and both groups were assessed before and after the intervention period by applying a focused attention test. The teachers were also interviewed regarding the changes perceived in the classroom. The results showed that the experimental group had a performance of attention in the test significantly superior to the control group, especially in the number of errors committed, referring to the improvement of the quality of
\end{abstract}


attention. In addition, the teachers highlighted the improvement in the focus and finalization of activities. In this way, it is reinforced that the use of digital games can contribute to digital inclusion and to the improvement of the learning conditions of the children.

Keywords: Digital games. Learning. Child education.

\section{Resumen}

La inclusión digital remite al acceso y al uso crítico y creativo de las tecnologías digitales en favor de la emancipación. Entre las tecnologías, se destacan los juegos digitales como alternativas para mejorar las condiciones de aprendizaje y de alfabetización digital. En este sentido, este estudio tiene como objetivo evaluar si una intervención pautada en el uso de juegos digitales en pequeños grupos en contexto escolar puede contribuir con el perfeccionamiento del desempeño de la atención, buscando garantizar mejores condiciones de aprendizaje e inclusión digital. Para ello, se realizó una investigación casi experimental de abordaje mixto con una muestra por conveniencia de 40 niños con edades entre 7 y 9 años, divididas en grupo experimental y control. El primer grupo participó en intervenciones semanales en pequeños grupos sobre la base del uso de juegos digitales y ambos grupos se evaluaron antes y después del período de las intervenciones mediante la aplicación de una prueba de atención concentrada. Las profesoras también fueron entrevistadas con respecto a los cambios percibidos en la sala. Los resultados revelaron que el grupo experimental tuvo un desempeño de la atención en la prueba significativamente superior al grupo control, especialmente en la cantidad de errores cometidos, remitiendo al mejoramiento de la calidad de la atención. Además, las profesoras destacaron la mejora en el foco y en la finalización de las actividades. De ese modo, se refuerza que el uso de los juegos digitales puede contribuir con la inclusión digital y con la mejora de las condiciones de aprendizaje de los niños.

Palabras clave: Juegos digitales. El aprendizaje. Educación Infantil. 


\section{Introdução}

A função emancipadora da escola se efetiva, sobretudo, na garantia de condições para que os sujeitos se desenvolvam e aprendam por meio da participação e vivência de experiências intencionalmente planejadas e diversificadas, que possibilitem a apropriação do conhecimento e sua inserção na sociedade. Nessa perspectiva, a diversificação curricular e o uso de diferentes recursos pedagógicos podem contribuir para que se fortaleça a função emancipadora. Isso porque a intervenção compensatória precisa "revestir-se de um modelo didático flexível e plural que permita atender às diferenças de origem [...] requer flexibilidade, diversidade e pluralidade metodológica e organizativa" (PÉREZ GÓMES, 1998, p. 23).

Este trabalho foca o uso das tecnologias digitais no contexto escolar como alternativa para melhorar as condições de aprendizagem e de letramento digital, o que remete a mecanismos de inclusão/exclusão constituídos por redes complexas de práticas sociais "que se apoiam, entrelaçam, contestam e modificam mútua e continuamente nas e por meio, virtude ou influência das TIC" (BUZATO, 2008, p. 328). Assim, parte-se do reconhecimento de que o uso das tecnologias digitais de maneira intencional na escola cria condições para o desenvolvimento de habilidades cognitivas fundamentais à aprendizagem, que podem ser aprimoradas por meio da experiência e da interação social, visando a superação, o letramento e a inclusão digital.

Dentre essas habilidades, destaca-se a atenção como condição fundamental à memória e à aprendizagem. Ao mesmo tempo, a falta de atenção em sala de aula é queixa recorrente dos professores e pode estar relacionada a uma ampla gama de dificuldades de aprendizagem. As questões vinculadas à atenção em sala de aula frequentemente manifestam-se nas dificuldades relacionadas ao esforço necessário para iniciar, organizar e finalizar uma tarefa, ao prestar e manter a atenção e ao controle das emoções, tornando difícil a aprendizagem de conceitos e habilidades (SMITH; STRICK, 2009). 
Diante disso, este estudo tem como objetivo avaliar se uma intervenção pautada no uso de jogos digitais em pequenos grupos em contexto extraclasse pode contribuir com o aprimoramento do desempenho da atenção, visando garantir melhores condições de aprendizagem e inclusão digital. Nesse sentido, descreve-se a intervenção realizada e apresentam-se os resultados obtidos por meio da realização de entrevistas com as professoras das crianças participantes da intervenção, além da aplicação de um teste de atenção concentrada em um estudo quase-experimental, realizado no contexto escolar, prevendo os grupos experimental e controle.

\section{Jogos digitais e o aprimoramento da atenção}

A atenção faz parte das funções executivas, especialmente relacionada ao controle inibitório (DIAMOND, 2013). As funções executivas integram diferentes habilidades cognitivas, exercem influência sobre os processos de aprendizagem e estão fortemente relacionadas com a capacidade adaptativa às demandas e às mudanças ambientais (MORTON, 2013; DIAMOND, 2013). Em síntese, a atenção refere-se à capacidade de se concentrar em informações relevantes e manter o processamento dessas informações, ignorando estímulos distratores (MATLIN, 2004; BUSCHMAN; MILLER, 2007; KORNRUMPF; SOMMER, 2015).

Muitos fatores podem influenciar essa habilidade, como a motivação, a experiência anterior na tarefa, o efeito do tempo na tarefa e a fonte de distração (BURNS et al, 2009; CARDOSO-LEITE; BAVELIER 2014). Além disso, a rede de atenção pode ser associada a fatores genéticos, apesar de que esse fator pode ser melhorado por intervenções durante o desenvolvimento (RUEDA et al, 2005). Ressalta-se, ainda, que a atenção é condição fundamental para a aprendizagem e a adequação dos comportamentos humanos, pois está envolvida na regulação de pensamentos e emoções, na manutenção do foco em uma atividade e na não 
apreensão de estímulos irrelevantes ou distratores (USCHMAN; MILLER, 2007; GAZZALEY; NOBRE, 2012; STERNBERG, 2008; DIAMOND, 2013).

No contexto escolar, revela-se relevante para aprendizagem, pois em sala de aula temos muitos estímulos, visuais, sonoros e ações, que concorrem para ser foco da atenção. Assim, a seleção das informações relevantes é importante, uma vez que nosso cérebro não tem a capacidade de armazenar todos os estímulos perceptivos que recebe constantemente (MATLIN, 2004; STERNBERG, 2008). A atenção relaciona-se intimamente à aprendizagem (GOTTLIEB, 2012; RAYMOND; O'BRIEN, 2009) e tem influência sobre o desempenho escolar (BORELLA; CARRETTI; PELEGRINA, 2010; DIAMOND; LEE, 2011). Assim, o treinamento de habilidades de atenção pode ser benéfico para a melhoria das habilidades acadêmicas, por estar relacionada à capacidade geral de aprendizagem (BANIQUED et al, 2013).

A partir do reconhecimento das contribuições que as tecnologias digitais podem oferecer e da importância da atenção como condição à aprendizagem, destaca-se o uso dos jogos digitais na escola como alternativa para a diversificação das atividades curriculares. Além disso, o reconhecimento de que as crianças possuem diferentes condições para a aprendizagem reforça a função compensatória da escola, o que tende a contribuir para atenuar os efeitos das desigualdades e preparar os alunos para se defenderem e atuarem no cenário social (PÉREZ GÓMEZ, 1998).

A proposição do uso de jogos digitais para o aprimoramento da capacidade de atenção constitui-se como uma intervenção que visa garantir melhores condições de aprendizagem, favorecendo, assim, a inclusão dos alunos. A utilização das tecnologias na educação tem impacto pedagógico sobre os processos de ensino e sobre os fatores que interferem na qualidade da aprendizagem (TRINDADE; MOREIRA, 2017).

Os jogos digitais possuem características como desafios crescentes e regras que estabelecem o que pode ou não ser feito, envolvendo o jogador na busca pela superação de suas habilidades para vencer o jogo, assemelhando-se ao processo de aquisição de conhecimentos e o desenvolvimento de habilidades (KIRRIEMUIR; MCFARLANE, 2004; 
PRENSKY, 2012). Quando inseridos no contexto escolar, os jogos fornecem condições de aprendizagem e desenvolvimento cognitivo. Arndt (2012) sugere que espaços de aprendizagem precisam favorecer diversos tipos de estímulo para garantir um bom desempenho dos alunos, já que as características estruturais influenciam a vontade de aprender, o bem-estar emocional e a sensação de segurança, características que possuem impacto significativo no desenvolvimento e na eficiência da aprendizagem.

As contribuições do uso dos jogos digitais relacionadas ao aprimoramento das funções cognitivas podem contribuir para que a escola desempenhe sua função emancipatória e inclusiva. Isso porque crianças com dificuldades relacionadas às funções executivas acabam se beneficiando mais do uso dos jogos digitais, o que oferece a possibilidade de alcançarem níveis próximos de seus pares, com a garantia de melhores condições de aprendizagem (DIAMOND, 2012).

A proposição das intervenções com jogos digitais apoia-se em resultados de estudos que têm indicado contribuições do seu uso para o aprimoramento das funções cognitivas em crianças em relação a diversos aspectos: memória de trabalho e atenção (THORELL et al, 2009; RUEDA; CHECA; CÓMBITA, 2012), flexibilidade e raciocínio (DOVIS et al, 2015) e funções executivas de maneira mais ampla (DIAMOND; LEE, 2011). Neste trabalho, propôs-se o uso da Escola do Cérebro como atividade extraclasse para crianças indicadas pelas professoras por identificarem alguma dificuldade em relação ao desempenho da atenção e por reconhecerem que poderiam se beneficiar das intervenções. A Escola do Cérebro é um aplicativo voltado para o público infantil que integra jogos digitais a uma base de dados para o exercício, o acompanhamento e a avaliação das habilidades cognitivas, especialmente a atenção, a capacidade de resolução de problemas e a memória de trabalho (RAMOS, 2014). 


\section{Metodologia}

O estudo desenvolvido caracteriza-se como uma pesquisa quase experimental (COHEN; MANION; MORRISON, 2007) de abordagem mista, utilizando-se instrumentos de coleta e procedimentos de análise tanto quantitativos como qualitativos (CRESWELL, 2010). A pesquisa foi realizada em duas escolas públicas, uma municipal e outra federal. As intervenções com os jogos aconteceram como atividade extraclasse, uma vez por semana, no contraturno.

\section{Participantes}

A amostra foi composta por conveniência, já que a seleção foi realizada a partir da indicação das professoras e coordenadoras pedagógicas da instituição, que resultou na participação de 40 crianças com idades entre 7 e 9 anos $(M=8,63 \pm 1,21)$, as quais frequentavam do $2^{\circ}$ ao $5^{\circ}$ ano do Ensino Fundamental. Além disso, sete professoras participaram das entrevistas após a realização das intervenções.

Os critérios para inclusão da amostra eram: a) estar matriculado regularmente no Ensino Fundamental; b) ter sido indicado pela professora, por reconhecer que as crianças tinham algum tipo de dificuldade de aprendizagem, relacionada especialmente à atenção, e que poderiam se beneficiar da participação no projeto; c) assinar o Termo de Consentimento Livre e Esclarecido (TCLE). Os critérios para exclusão foram: a) falta de colaboração/presença nas etapas de avaliação ou intervenção; b) comorbidades neuropsiquiátricas ou deficiência mental.

Os participantes foram organizados em dois grupos, um participante, o qual frequentou os atendimentos semanais, e outro de controle, que apenas foi avaliado antes e depois do período das intervenções com os mesmos instrumentos. Foram incluídas no grupo de participantes as crianças que se dispuseram a frequentar os atendimentos oferecidos no contraturno. O grupo controle foi composto pelas crianças, também 
indicadas pelas professoras, mas cujas famílias não conseguiram viabilizar a participação.

\section{Instrumentos e materiais da pesquisa}

As intervenções realizadas pautaram-se no uso de jogos digitais cognitivos da Escola do Cérebro. Estão disponíveis oito jogos digitais que são classificados conforme a intensidade de uso de três habilidades cognitivas: memória de trabalho, atenção e resolução de problemas.

O acesso aos jogos na Escola Municipal foi realizado por meio do uso de computadores da sala de informática, e, na Escola Federal, por tablets Android. A avaliação das crianças no início e no final das intervenções, para mensurar as mudanças no desempenho da atenção, foi realizada por meio da aplicação do Teste de Atenção Concentrada — D2. Este é um teste psicométrico, validado para a população brasileira pelo Sistema de Avaliação de Testes Psicológicos do Conselho Federal de Psicologia Brasileiro, cujas propriedades psicométricas avaliam a atenção sustentada. Seu escore é formado a partir da habilidade do indivíduo para selecionar uma fonte de informação, ignorando os distratores, e conseguir mantê-la no decorrer do tempo. Os dados extraídos para análise foram: escore bruto, erros e escore líquido (subtração do número de erros do escore bruto) (BRICKENKAMP, 2000).

Por fim, utilizou-se um roteiro de entrevista semiestruturado, aplicado ao final das intervenções com as professoras das crianças participantes, com o objetivo de estabelecer um diálogo sobre as percepções acerca da identificação de mudanças comportamentais e de desempenho escolar das crianças. De modo geral, as questões buscavam investigar as mudanças observadas. 


\section{Procedimentos}

A pesquisa foi submetida e aprovada pelo Comitê de Ética em Pesquisa da universidade, à qual os pesquisadores são vinculados (CAEE 67638216.5.0000.0121). A partir da indicação feita pelas professoras e coordenadoras, as crianças foram convidadas a participar do atendimento focal que ocorre uma vez por semana, por 50 minutos, em grupos de 2 a 4 crianças, com base no uso de jogos cognitivos.

Além do uso dos jogos, o atendimento caracteriza-se por sua estrutura inspirada na terapia cognitivo-comportamental; entretanto, foram feitas adaptações ao contexto educacional, focando-se na capacidade de atenção. Nos atendimentos, inicialmente, propôs-se às crianças um exercício de autopercepção, por meio do uso de uma escala de atenção, na qual a criança pinta o smile que mais representa sua atenção naquele momento. A escala varia de 1 a 5 e ilustra smiles que representam expressões da mais sonolenta para última, focada na leitura.

Após a avaliação da capacidade de atenção, estabeleciam-se os combinados sobre o que seria jogado, reforçando as regras e a negociação. Então, o desenvolvimento envolvia a interação com os jogos disponibilizados na Escola do Cérebro, normalmente focando no uso de um dos jogos. Em seguida, propunha-se a avaliação do comportamento das crianças em grupo, por meio do uso de uma tabela de reforçamento, contemplando as atitudes, a atenção, o compromisso e a interação social durante o atendimento (RAMOS, 2014). Por fim, uma tarefa era passada para cada criança, cujo retorno é avaliado no item "compromisso" da semana seguinte.

Nas primeiras e últimas sessões do atendimento focal, as crianças foram avaliadas com base na aplicação do Teste de Atenção Concentrada - D2. Essa avaliação foi repetida ao final dos atendimentos, que duraram aproximadamente 4 meses, resultando em um média de participação de 13 sessões, variando de 7 a 25 sessões (desvio padrão = 6,41). Após o período de desenvolvimento das atividades de intervenção, as professoras das crianças participantes foram entrevistadas, procurando 
identificar mudanças percebidas que pudessem ser relacionadas às atividades desenvolvidas.

\section{Análise dos dados}

Os dados coletados por meio da aplicação e correção dos testes foram tabulados com o software Excel para criação da base de dados. Após a organização das informações, os dados foram analisados no software SPSS (Statistical Package for the Social Sciences), versão 24, para a análise estatística.

A análise quantitativa dos dados teve início a partir da averiguação da normalidade dos dados; por meio do teste Shapiro-wilk, procedeu-se à realização dos testes estatísticos para comparar os resultados antes e depois do desempenho no teste, procurando indicadores sobre a influência das intervenções sobre o desempenho das crianças. Dada a normalidade dos dados, foram formados dois grupos denominados "Intervenção" (grupo controle e grupo experimental) e "Período" (antes e depois da intervenção). Os dados foram submetidos ao teste ANOVA para medidas repetidas, tendo a variável "Intervenção" como fator entre indivíduos, e a variável "período" como fator de comparação interindividual. Para ambos os testes, foi considerado o intervalo de confiança de $95 \%$.

Os dados qualitativos obtidos por meio da realização das entrevistas com as professoras foram transcritos e analisados com base na análise de conteúdo de Bardin (1977), realizando-se a pré-análise pela leitura flutuante das transcrições para a identificação das categorias e dos indicadores, bem como preparação do material para análise. A análise e codificação com base nos indicadores foi realizada com o uso do software NVivo. Por fim, realizou-se o tratamento dos resultados, o que possibilitou a ocorrência de inferências e interpretações. 


\section{Resultados}

A ANOVA para medidas repetidas verificou o efeito do "Grupo" (grupo controle vs. grupo experimental), "Período" (antes da intervenção vs. após a intervenção) e a interação destas variáveis no desempenho atencional (escore bruto, número de erros e escore líquido) mensurado pelo teste D2 (Tabela 1). A análise sugere que a variável "Grupo" apresenta efeito significativo no escore bruto $(F=9,55, \mathrm{p}=0,004)$ e líquido $(F=6,02$, $\mathrm{p}=0,01$ ) do teste de atenção $\mathrm{D} 2$, cujos valores do grupo controle (média do escore bruto $=331,67$ e média do escore líquido $=296,02)$ são superiores ao do grupo experimental (média do escore bruto $=270,57$ e média do escore líquido $=240,72)$. Resultado semelhante foi observado para a variável "Período", em que se observaram valores superiores do escore bruto $(\mathrm{F}=34,53, \mathrm{p}=0.0001)$ e líquido $(\mathrm{F}=69,47, \mathrm{p}=0,0001)$ nas avaliações após a intervenção (média do escore bruto $=327,32$ e média do escore líquido = 297,78), quando comparados aos valores iniciais (média do escore bruto = 274,92 e média do escore líquido $=296,02)$, sugerindo que ambos os grupos melhoraram a performance após a intervenção.

A interação entre as variáveis "Grupo" e "Período" nas medidas de desempenho do teste D2 (Figura 2) sugere que o grupo controle $(\mathrm{M}=370,30)$ apresentou valores maiores do escore bruto $(\mathrm{F}=7,76$, $\mathrm{p}=0,0008)$, quando comparado ao grupo experimental $(\mathrm{M}=284,35)$. Por outro lado, observou-se que o grupo controle apresentou um número de erros superior $(M=40,10)$ quando comparado ao experimental $(M=19,00$, $\mathrm{F}=10,25, \mathrm{p}=0,003)$. Estes resultados sugerem que, embora o grupo controle tenha um número elevado de respostas, também apresentou um número superior de erros, quando comparado ao experimental. Não foi observada diferença estatística significativa para a interação destas variáveis no escore líquido $(\mathrm{F}=1,83, \mathrm{p}=0,18)$. 
Tabela 1 - Resultado da ANOVA para medidas repetidas para a comparação das variáveis "Grupo" e "Período" nas medidas de desempenho do teste D2.

\begin{tabular}{|c|c|c|c|c|c|c|}
\hline \multirow[t]{2}{*}{ D2 - Escore Bruto } & \multicolumn{2}{|c|}{ Grupo Controle $(\mathrm{N}=20)$} & \multicolumn{2}{|c|}{$\begin{array}{l}\text { Grupo Experimental } \\
\qquad(\mathrm{N}=20)\end{array}$} & \multirow[t]{2}{*}{$F$} & \multirow[t]{2}{*}{$\mathbf{p}$} \\
\hline & Antes & Após & Antes & Após & & \\
\hline Grupo* & \multicolumn{2}{|c|}{$331,67 \pm 82,59$} & \multicolumn{2}{|c|}{$270,57 \pm 50,25$} & 9,55 & 0,004 \\
\hline Período* & \multicolumn{2}{|c|}{$274,92 \pm 71,28$} & \multicolumn{2}{|c|}{$327,32 \pm 79,44$} & 34,53 & 0.0001 \\
\hline Interação* & $\begin{array}{r}293,05 \\
\pm 87,77\end{array}$ & $\begin{array}{c}370,30 \pm \\
77,42\end{array}$ & $\begin{array}{r}256,80 \\
\pm 45,09\end{array}$ & $\begin{array}{r}284,35 \\
\pm 55,43\end{array}$ & 7,76 & 0,008 \\
\hline \multicolumn{7}{|l|}{ D2 - Erros } \\
\hline Grupo & \multicolumn{2}{|c|}{$35,65 \pm 35,79$} & \multicolumn{2}{|c|}{$28.85 \pm 23.28$} & 0,42 & 0,519 \\
\hline Período & \multicolumn{2}{|c|}{$35,95 \pm 27,81$} & \multicolumn{2}{|c|}{$29,55 \pm 36,79$} & 1,79 & 0,118 \\
\hline Interação* & $\begin{array}{c}31,20 \\
\pm 26,24\end{array}$ & $\begin{array}{c}40,10 \\
\pm 47,35\end{array}$ & $\begin{array}{c}40,70 \\
\pm 29,18\end{array}$ & $\begin{array}{c}19,00 \\
\pm 17,39\end{array}$ & 10,25 & 0,003 \\
\hline \multicolumn{7}{|l|}{ D2 - Escore Liquído } \\
\hline Grupo* & \multicolumn{2}{|c|}{$296,02 \pm 91.51$} & \multicolumn{2}{|c|}{$240,72 \pm 51,35$} & 6,02 & 0,019 \\
\hline Período* & \multicolumn{2}{|c|}{$238,98 \pm 78,60$} & \multicolumn{2}{|c|}{$297,78 \pm 79,37$} & 69,47 & 0,0001 \\
\hline Interação & $\begin{array}{r}261,85 \\
\pm 97,22\end{array}$ & $\begin{array}{l}330,20 \\
\pm 85,80\end{array}$ & $\begin{array}{r}216,10 \\
\pm 46,15\end{array}$ & $\begin{array}{r}265,35 \\
\pm 56,57\end{array}$ & 1,83 & 0,184 \\
\hline
\end{tabular}

Legenda: "Grupo" representa o efeito da comparação entre o grupo controle e o experimental. "Período" representa o efeito da comparação entre os valores da avaliação antes e após a intervenção. "Interação" representa o efeito da interação entre as variáveis "Grupo" e "Período". As variáveis destacadas com "*" apresentaram $p<0.05$ para a ANOVA de medidas repetidas.

Fonte: Os autores. 
Figura 1 - Efeito das variáveis "Grupo" e "Período" ao escore bruto (A), número de erros (B) e escore líquido (C) do teste D2

A

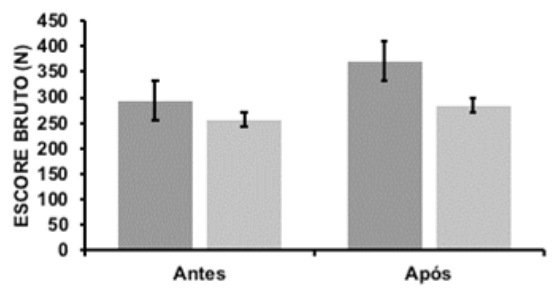

B

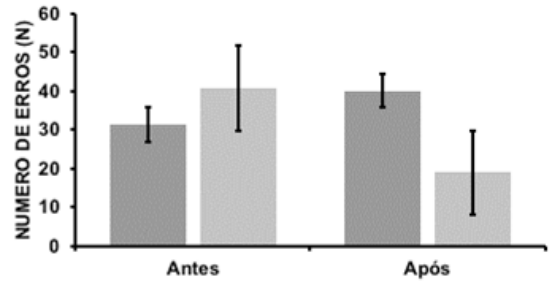

C

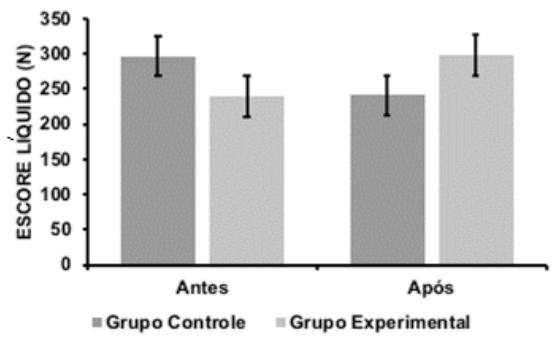

Fonte: Os autores.

Os resultados obtidos na entrevista das professoras com relação ao grupo experimental reforçaram as melhoras em relação à atenção. As professoras relataram ter percebido mudanças que indicam melhoras na atenção em 15 das 20 crianças que compuseram o grupo participante.

Essas melhoras foram observadas em relação a comportamentos como a manutenção do foco, a diminuição na frequência de comportamento que revelam distração, a finalização das atividades propostas em sala e a maior persistência na resolução de problemas e na realização das atividades. No Gráfico 1, pode-se observar a frequência de codificação dos comportamentos indicativos de melhora da atenção em sala de aula, categorizados a partir da transcrição das falas das entrevistas com as professoras. 
Gráfico 1 - Codificação das categorias de atenção da transcrição das entrevistas com as professoras do grupo experimental.

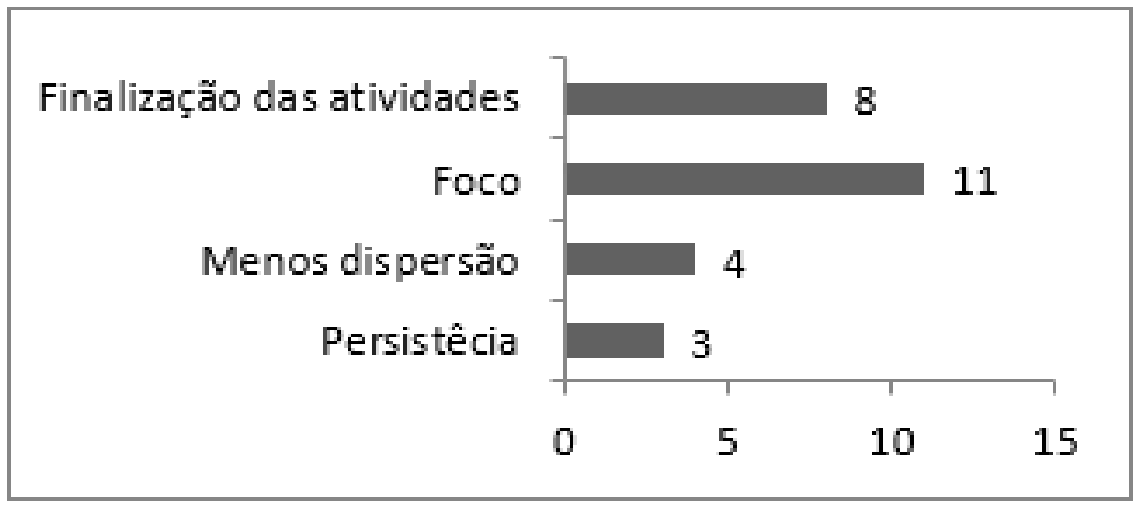

Fonte: os autores.

A categoria relacionada a comportamentos que indicam melhora na atenção mais citada pelas professoras foi o foco, que correspondeu a 42,31\% da frequência de codificação. Essa categoria foi identificada e codificada a partir de transcrições como: "Mais atencioso nas atividades" (Professora 3), "quando se organizava passou a conseguir falar; melhorou o foco e a atenção" (Professora 7) e "conseguiu manter mais o foco nas atividades de matemática" (Professora 4).

Outra categoria relacionada à atenção foi o relato de menos dispersão em sala, que foi codificada em transcrições como: "Não fica mais na turma da bagunça, não brincou mais com cartinha" e "antes não se concentrava, se distraía com brincadeiras em sala e melhorou" (Professora 1). Essa categoria registrou a frequência de 15,38\%.

A finalização das atividades em sala, como categoria indicativa da melhora de atenção em sala de aula, foi a segunda mais frequente, com 30,77\% das codificações. Essa categoria relaciona-se a transcrições como: "passou a concluir mais as atividades" (Professora 5), "passou a concluir as atividades, antes não concluía e agora faz todas mesmo que errado" e "agora consegue terminar as atividades" (Professora 2). 
Incluímos ainda a persistência como indicador de melhora da atenção, resultando em $11,54 \%$ das frequências e inclui a codificação de falas como "Teve uma mudança, tornou-se mais persistente" e "Antes tinha menos persistência, agora tenta mais".

Algumas falas transcritas remetem a mais de uma categoria, reforçando as mudanças percebidas pela professora em sala. Uma das professoras, ao descrever sua percepção de uma das crianças, afirma: "agora consegue se manter mais atenta, não mais participativa, mas menos dispersa. Antes ficava muito entretida com outras coisas. Começou a finalizar as atividades mais no tempo da turma. Melhorou, mas ainda precisa melhorar mais" (Professora 3). Outra professora também destaca a mudança em outra criança: "Foi da água para $o$ vinho, mudou muito desde o início do ano. Falta bastante. Deu um salto na aprendizagem, mas agora escreve, lê e no começo do ano não conhecia as letras. Continua inquieto, brigão. Ele consegue focar quando está fazendo uma atividade. Foi surpreendente a melhora em termos de aprendizagem" (Professora 6).

\section{Discussão}

Os resultados descritos, tanto relacionados ao desempenho da atenção medida pela aplicação dos testes como do relato das professoras, sugerem que a proposição do uso de jogos digitais como atividade complementar à rotina escolar pode contribuir com aprimoramento do desempenho atencional de alunos do Ensino Fundamental.

De modo geral, ambos os grupos apresentaram um aumento nos escores na segunda avaliação, porém o grupo participante obteve uma melhora mais acentuada. Embora exista uma interação entre as variáveis "grupo" e "período", o que sugere uma diferença entre a melhora da atenção para o grupo de treinamento quando comparado ao controle, o grupo controle também apresentou um aprimoramento de desempenho quando se comparam os resultados das avaliações de atenção pré e pós. Assim, é preciso salientar que essa melhora pode estar relacionada ao fator teste e reteste, uma vez que as crianças já haviam realizado a tarefa anteriormente. 
Apesar disso, a comparação entre o desempenho da atenção entre os dois grupos revelou que os alunos que participaram da intervenção com jogos digitais tiveram um aumento mais significativo nos índices de escore bruto e líquido, bem como diminuíram o número de erros. E esse melhor desempenho da atenção é reforçado pela percepção das professoras das crianças em sala de aula.

Os resultados obtidos corroboram com vários estudos que investigaram os efeitos dos jogos digitais no desempenho cognitivo (BANIQUED et al, 2013; DIAMOND; LEE 2011; HOMER et al, 2018). Dentre os estudos que apresentam diferentes desenhos de intervenção, há estudos realizados em laboratório propondo o treinamento individual (NOUCHI et al., 2013). Outros envolvem a interação diária de jogo digital, obtendo melhoras no desempenho cognitivo após dez semanas de treinamento diário de 20 minutos (MILLER; ROBERTSON, 2010). Homer et al, (2018) também revelam melhora na flexibilidade cognitiva e na atenção seletiva após o treinamento diário de jogo digital (20 minutos) durante seis semanas.

Os resultados deste estudo sugerem que o treinamento cognitivo por meio da interação com jogos digitais pode beneficiar o desempenho da atenção, mesmo quando realizado em pequenos grupos e intervenções semanais. No entanto, permanecem em aberto as contribuições sobre outros processos cognitivos envolvidos, como a memória de trabalho e a capacidade de resolução de problemas, uma vez que esta pesquisa apenas focou os processos de atenção. Outros processos cognitivos tendem também a apresentar melhorias, pois alguns estudos indicam que a interação com jogos digitais no ambiente escolar melhora as habilidades escolares em geral (ROSAS et al, 2003), a memória de trabalho, a capacidade de planejamento, a tomada de decisão e a velocidade de processamento (CARDOSO-LEITE; BAVELIER, 2014).

As evidências encontradas sugerem que o treinamento cognitivo por jogos digitais pode ser uma alternativa para a diversificação das atividades escolares, para a melhora das condições de aprendizagem das crianças, considerando a importância da atenção para o processo de aprendizagem (RAYMOND; O’BRIEN, 2009; GOTTLIEB, 2012; BANIQUED et al, 2013), 
especialmente no espaço escolar, onde a criança precisa se apropriar de conteúdos em meio a um ambiente com inúmeros distratores.

Destaca-se que o melhor desempenho no teste de atenção concentrada, e a percepção das professoras em relação aos comportamentos apresentados em sala, revelam-se indicativos de que a melhora da atenção proporcionada pela interação com os jogos digitais pode ser transferida para outras atividades, incluindo as escolares. Estudos sugerem que a transferência de habilidades cognitivas ocorre para tarefas que compartilham algumas características semelhantes à tarefa, como observado por Oei e Patterson (2013), ao proporem o treinamento cognitivo utilizando jogos de diferentes categorias e observarem melhoras específicas no desempenho cognitivo de acordo com o jogo. Outro estudo, desenvolvido por Thorell et al (2009), que avaliou o treinamento específico de memória de trabalho, observou melhoras em tarefas de memória de trabalho e de atenção, porém o grupo de treinamento de controle inibitório não generalizou suas habilidades de treinamento para outros testes de controle inibitório ou outras tarefas.

As análises consideram o efeito "período", que vale ser destacado ao considerar-se que os participantes são crianças que apresentam uma curva de desenvolvimento cognitivo maturacional, (GARON; BRYSON; SMITH et al, 2008) e que a própria rotina escolar, com suas atividades e regras, influencia o desenvolvimento de funções executivas (DIAMOND; LEE, 2011).

Reforça-se que o enriquecimento ambiental, por meio da inserção das tecnologias digitais, incluindo os jogos digitais, pode ajudar também na inclusão de alunos com problemas de aprendizagem mais graves, como dislexia (HARRAR et al, 2014) e TDAH (DE LA GUIA et al, 2015; DOVIS et al, 2015). Assim, há evidências de que a integração de tecnologias, especialmente dos jogos digitais, pode ser alternativa à diversificação curricular e contribuir para garantir melhores condições de inclusão, reforçando a função emancipadora da escola. Diante disso, "é fundamental que os próprios professores saibam apropriar-se das vantagens destas tecnologias, e as usem na criação de novos ambientes de aprendizagem, mais motivadores, mais estimulantes" (TRINDADE; MOREIRA, 2017, p. 101), visando o desenvolvimento de habilidades importantes para atuação na sociedade contemporânea. 
Apesar das evidências que podem ser associadas à intervenção proposta e avaliada no estudo para a melhora do desempenho da atenção, podem-se reconhecer algumas limitações, como, por exemplo, o fato de não se ter controle sobre a interação com jogos digitais fora da escola (p. ex. em casa) e a aplicação dos testes ocorrer em momentos pontuais, em que fatores como disposição e motivação, por exemplo, podem influenciar o desempenho. Por isso, a busca pela triangulação entre os resultados dos testes e a entrevista com as professoras revela-se importante para reforçar as melhoras em relação à atenção. Finalmente, também seria necessário avaliar outros processos cognitivos para avaliar se existem benefícios para outras habilidades relacionadas à capacidade de aprendizagem, especialmente porque, nas entrevistas, as professoras relatam melhoras em relação a outras habilidades, que também têm influência sobre a aprendizagem.

\section{Considerações finais}

Os resultados descritos evidenciam que a intervenção pautada no uso de jogos digitais, realizada em pequenos grupos no contexto extraclasse, contribuiu para o aprimoramento do desempenho da atenção, pois o grupo experimental teve um desempenho significativamente superior quando comparado ao grupo controle, especialmente em relação à diminuição da quantidade de erros, o que indica uma melhora na qualidade de atenção. As professoras também reforçam, a partir da observação das crianças, a melhoria da manutenção do foco, da finalização das atividades, da diminuição da dispersão e da manifestação de maior persistência. Essas mudanças tendem a contribuir para garantir melhores condições de aprendizagem e a inclusão digital das crianças.

A partir disso, destaca-se o uso dos jogos digitais por suas características que incluem os desafios, as regras, a narrativa e o feedback para criar experiências de aprendizagem que favoreçam o aprimoramento das habilidades cognitivas. Assim, temos o uso da inclusão e das tecnologias digitais voltado para o desenvolvimento dos alunos e para garantir melhores condições 
de aprendizagem, favorecendo, consequentemente, sua inserção social e a superação das dificuldades no contexto escolar. Apesar de o estudo voltar-se para o aprimoramento da capacidade de atenção, outros aspectos poderiam ser explorados em trabalhos futuros, como o papel da motivação relacionada ao uso das tecnologias e dos jogos. Além disso, a mediação realizada pelos pesquisadores pode ter influenciado a melhoria do desempenho. Cabe, também, questionar até que ponto o desempenho da atenção obtido na tarefa proposta pode ser transferida para outras atividades no contexto escolar.

De qualquer modo, evidenciamos contribuições significativas para as crianças participantes, o que tende a contribuir efetivamente com o processo de aprendizagem, por meio da proposição de atividades lúdicas e baseadas em tecnologias digitais e, por consequência, na inclusão digital, o que diversificou as estratégias pedagógicas e os recursos utilizados na escola.

\section{Referências}

ARNDT, P. A. Design of learning spaces: emotional and cognitive effects of learning environments. Mind, Brain, and Education, v. 6, n. 1, p. 41-48, mar. 2012.

BANIQUED, P. L. et al. Selling points: what cognitive abilities are tapped by casual video games? Acta psychologica, v. 142, n. 1, p. 74-86, 2013.

BARDIN, L. Análise de conteúdo. Lisboa: Editora Edições, 1977.

BORELLA, E.; CARRETTI, B.; PELEGRINA, S. The specific role of inhibition in reading comprehension in good and poor comprehenders. Journal of Learning disabilities, v. 43, n. 6, p. 541-552, 2010.

BRICKENKAMP, R. Teste D2 Atenção Concentrada. 2. ed. São Paulo: CETEP, 2000. BURNS, N. R.; NETTELBECK, T.; MCPHERSON, J. Attention and intelligence: a factor analytic study. Journal of Individual Differences, v. 30, n. 1, p. 44-57, 2009.

BUSCHMAN, T. J.; MILLER, E. K. Top-down versus bottom-up control of attention in the prefrontal and posterior parietal cortices. Science, v. 315, n. 5820, p. 1860-1862, 2007. 
BUZATO, M. El K. Inclusão digital como invenção do quotidiano: um estudo de caso. Revista Brasileira de Educação, v. 13, n. 38, p. 325-413, 2008.

CARDOSO-LEITE, P.; BAVELIER, D. Video game play, attention, and learning: how to shape the development of attention and influence learning? Current opinion in neurology, v. 27, n. 2, p. 185-191, 2014.

COHEN, L.; MANION, L.; MORRISON, K. Research methods in education. London and New York: Routledge, 2007.

CRESWELL, J. W. Projeto de pesquisa: métodos qualitativos, quantitativos e mistos. 3. ed. Porto Alegre, Artmed, 2010.

DE LA GUÍA, E.; LOZANO, M. D.; PENICHET, V. M. R. Educational games based on distributed and tangible user interfaces to stimulate cognitive abilities in children with ADHD. British Journal of Educational Technology, v. 46, n. 3, p. 664-678, 2015.

DIAMOND, A. Executive functions. Annual Review of Psychology, v. 64, p. 135-68, 2013.

DIAMOND, A. Activities and programs that improve children's executive functions. Current directions in psychological science, v. 21, n. 5, p. 335-341, 2012.

DIAMOND, A.; LEE, K. Interventions shown to aid executive function development in children 4 to 12 years old. Science, v. 333, n. 6045, p. 959-964, 2011.

DOVIS, S., et al. Improving executive functioning in children with ADHD: training multiple executive functions within the context of a computer game. A randomized double-blind placebo controlled trial. PloS one, v. 10, n. 4, p. e0121651, 2015.

GARON, N.; BRYSON, S. E.; SMITH, I. M. Executive function in preschoolers: a review using an integrative framework. Psychological bulletin, v. 134, n. 1, p. 31, 2008.

GAZZALEY, A.; NOBRE, A. C. Top-down modulation: bridging selective attention and working memory. Trends in cognitive sciences, v. 16, n. 2, p. 129-135, 2012.

GOTTLIEB, J. Attention, learning, and the value of information. Neuron, v. 76, n. 2, p. 281-295, 2012. 
HARRAR, V. et al. Multisensory integration and attention in developmental dyslexia. Current Biology, v. 24, n. 5, p. 531-535, 2014.

HOMER, B. D. et al. Improving high school students' executive functions through digital game play. Computers \& Education, v. 117, p. 50-58, 2018.

KIRRIEMUIR, J.; MCFARLANE, A. Literature review in games and learning. Bristol: Futurelab, 2004. Disponível em: <https://hal.archives-ouvertes.fr/hal00190453/document >. Acesso em: 27 jan. 2018.

KORNRUMPF, B.; SOMMER, W. Modulation of the attentional span by foveal and parafoveal task load: an ERP study using attentional probes. Psychophysiology, v. 52, n. 9, p. 1218-1227, 2015.

MATLIN, M. W. Psicologia cognitiva. 5. ed. Rio de Janeiro: LTC, 2004.

MILLER, D. J.; ROBERTSON, D. P. Using a games console in the primary classroom: effects of 'Brain Training'programme on computation and self-esteem. British Journal of Educational Technology, v. 41, n. 2, p. 242-255, 2010.

MORTON, B. J. Enciclopédia sobre o desenvolvimento na primeira infância. Canadá: University of Western Ontario, 2013.

NOUCHI, R., et al. Brain training game boosts executive functions, working memory and processing speed in the young adults: a randomized controlled trial. PLoS ONE, v. 8, n. 2, 2013.

OEI, A. C.; PATTERSON, M. D. Enhancing cognition with video games: a multiple game training study. PLoS ONE, v. 8, n. 3, 2013.

PÉREZ GÓMEZ, A. I. As funções sociais da escola: da reprodução à reconstrução crítica do conhecimento e da experiência. In: GIMENO SACRISTÁN, J.; PÉREZ GÓMEZ, A. I. Compreender e transformar o ensino. 4. ed. Porto Alegre: ArtMed, 1998. p. 13-26. PRENSKY, M. Aprendizagem baseada em jogos digitais. 1. ed. São Paulo: SENAC, 2012. RAMOS, D. K. Cognoteca: uma alternativa para o exercício de habilidades cognitivas, emocionais e sociais no contexto escolar. Revista da FAEEBA-Educação e Contemporaneidade, v. 23, n. 41, 2014. 
RAYMOND, J. E.; O'BRIEN, Jennifer L. Selective visual attention and motivation: the consequences of value learning in an attentional blink task. Psychological Science, v. 20, n. 8, p. 981-988, 2009.

ROSAS, R., et al. Beyond Nintendo: design and assessment of educational video games for first and second grade students. Computers \& Education, v. 40, n. 1, p. 71-94, 2003.

RUEDA, M. R. et al. Training, maturation, and genetic influences on the development of executive attention. Proceedings of the national Academy of Sciences of the United States of America, v. 102, n. 41, p. 14931-14936, 2005.

RUEDA, M. R.; CHECA, P.; COMBITA, L. M. Enhanced efficiency of the executive attention network after training in preschool children: immediate changes and effects after two months. Developmental cognitive neuroscience, v. 2, p. S192-S204, 2012.

SMITH, C.; STRICK, L. Dificuldades de aprendizagem de A a Z: guia completo para educadores e pais. Penso Editora, 2009.

STERNBERG, R. Psicologia cognitiva. 4. ed. Porto Alegre: Artmed, 2008.

THORELL, L. B., et al. Training and transfer effects of executive functions in preschool children. Developmental science, v. 12, n. 1, p. 106-113, 2009.

TRINDADE, S. D.; MOREIRA, J. A. Competências de aprendizagem e tecnologias digitais. In: MOREIRA, J. A.; VIEIRA, C. P. (Coord.). eLearning no ensino superior. Coimbra: CINEP/IPC, 2017.

Recebido: 31/07/2018

Received: 07/31/2018

Recibido: 31/07/2018

Aprovado: 10/09/2018 Approved: 09/10/2018 Aprobado: 10/09/2018 